\title{
Analytic representation of
}

\section{critical equations of state}

\author{
Arnold Neumaier \\ Fakultät für Mathematik, Universität Wien \\ Oskar-Morgenstern-Platz 1, A-1090 Wien, Austria \\ email: Arnold.Neumaier@univie.ac.at \\ WWW: http://wWw.mat.univie.ac.at/ neum/
}

arXiv: 1401.0291

October 24, 2018

\begin{abstract}
A new form is proposed for equations of state (EOS) of thermodynamic systems in the 3-dimensional Ising universality class. The new EOS guarantees the correct universality and scaling behavior close to critical points and is formulated in terms of the scaling fields only - unlike the traditional Schofield representation, which uses a parametric form.

Close to a critical point, the new EOS expresses the square of the strong scaling field $\Sigma$ as an explicit function $\Sigma^{2}=D^{2 e_{-1}} W\left(D^{-e_{0}} \Theta\right)$ of the thermal scaling field $\Theta$ and the dependent scaling field $D>0$, with a smooth, universal function $W$ and the universal exponents $e_{-1}=\delta /(\delta+1), e_{0}=1 /(2-\alpha)$. A numerical expression for $W$ is derived, valid close to critical points.

As a consequence of the construction it is shown that the dependent scaling field can be written as an explicit function of the relevant scaling fields without causing strongly singular behavior of the thermodynamic potential in the one-phase region.

Augmented by additional scaling correction fields, the new EOS also describes the state space further away from critical points. It is indicated how to use the new EOS to model multiphase fluid mixtures, in particular for vapor-liquid-liquid equilibrium (VLLE) where the traditional revised scaling approach fails.
\end{abstract}

Keywords: analytic representation, complete scaling, corrections to scaling, critical equation of state, critical points, fluid mixtures, Ising universality class, order parameter, scaling fields, universality, vapor-liquid-liquid equilibrium, VLLE 


\section{Contents}

1 Introduction $\quad 2$

$\begin{array}{llr}2 & \text { Critical scaling } & 3\end{array}$

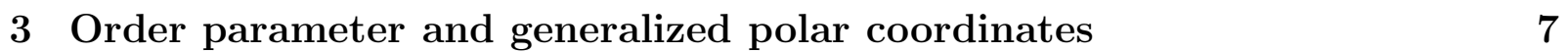

4 A $\Sigma$-explicit critical EOS $\quad 9$

5 A $D$-explicit formulation $\quad 11$

6 A global $\Sigma$-explicit scaling equation of state $\quad 13$

7 Phenomenological scaling models for fluid mixtures $\quad 15$

8 A multiphase critical equation of state $\quad 16$

9 Conclusion $\quad 18$

$\begin{array}{ll}\text { A Universality in generalized polar coordinates } & 19\end{array}$

B Comparison with data from the literature $\quad 22$

$\begin{array}{ll}\text { References } & 23\end{array}$

\section{Introduction}

This paper owes its existence to the desirability of a multi-phase, multi-component equation of state for fluids and fluid mixtures, valid over the whole fluid regime, with the property that, close to critical points, plait points, and consolute points, the correct universality and scaling behavior is guaranteed. The search for such an equation of state lead to the content of the present paper - the discovery that one can write the equation of state of any system with a critical point in the (3-dimensional) Ising universality class in a natural analytic form that improves upon traditional formulations. 
Equations of state valid over the whole fluid range must account for the singular behavior of various thermodynamic observables close to critical points (for pure fluids a liquid-vapor critical point, for fluid mixtures plait points at liquid-vapor equilibrium and consolute points at liquid-liquid equilibrium) and of the universal, substance independent power laws with which certain thermodynamic quantities scale near the critical point; cf. the fairly recent survey by Sengers \& Shanks [54].

Because of universality, the details of the microscopic models of a fluid are nearly irrelevant for determining the universal features near the critical point; thus highly simplified models may be used. The simplest is the lattice gas, mathematically equivalent to the Ising model for magnetization; ${ }^{1}$ see Pelissetto \& Vicari $[46,(1.3)]$. One therefore says that fluids and fluid mixtures belong to the (3-dimensional) Ising universality class. ${ }^{2}$ Initially thought to apply to fluids composed of molecules with short range forces only, it is now believed that ionic liquids, interacting with long range Coulombic forces, also belong to this universality class; see GutKowski et al. [27], SchröEr [53].

In the following, Section 2 summarizes the background on critical scaling and universality in general, and special properties of the Ising universality class. Section 3 introduces a generalization of the traditional Schofield representation (SCHOfIELD [52]) that, close to a critical point, eliminates the singularities by a parameterization in terms of generalized polar coordinates. Based on this, Section 4 derives the new $\Sigma$-explicit EOS close to a critical point. This form of the EOS was inspired by some explicitly solvable statistical mechanics models discussed by Fisher \& Felderhof $[18,19]$. Section 5 proves the existence of an analytic $D$-explicit EOS. Section 6 extends the new $\Sigma$-explicit EOS to states further away from the critical point, by incorporation scaling correction fields. Section 7 reviews phenomenological scaling models for fluid mixtures, and Section 8 proposes a phenomenological critical scaling model for multiphase fluid mixtures. Section 9 summarizes the main results. The appendices provide additional material with numerical details.

Acknowledgments. I'd like to thank Jan Stengers for pointing out an error in an intermediate version of this paper. I also acknowledge with pleasure several discussions with Ali Baharev, Waltraud Huyer, and Hermann Schichl on earlier versions of this manuscript, which lead to significant improvements.

\section{Critical scaling}

A thermodynamic field is a function of pressure $P$, absolute temperature $T$, and the chemical potential $\mu_{i}$ of each pure component $i$ in the mixture. For a fluid with $C$ components, the physically realizable thermodynamic states form a $(C+1)$-dimensional

\footnotetext{
${ }^{1}$ As a result, much of the statistical mechanics literature on critical scaling is written in a "magnetic" terminology. Most of this language is inappropriate in a fluid mixture context. Hence we choose here an independent notation and indicate at times alternative traditional notation in footnotes.

${ }^{2}$ For a recent verification in case of the Lennard-Jones fluid see WatanaBE et al. [58].
} 
manifold (with singularities) in the $(C+2)$-dimensional $(P, T, \mu)$-space, hence are described by an equation of state (EOS) relating $P, T$, and $\mu$.

The critical behavior of fluid (or solid) mixtures is believed to be characterized by the existence of two relevant scaling fields, ${ }^{3}$ the strong scaling field $\Sigma$ and the thermal scaling field $\Theta$.

The strong scaling field $\Sigma$ and the thermal scaling field $\Theta$ have a clear physical meaning: For $\Theta \leq 0$, the system is in a lower density phase if $\Sigma>0$, in a higher density phase if $\Sigma<0$, and has two coexistent lower and higher density phases if $\Sigma=0$. At $\Theta<$ $0=\Sigma$, we have a first-order phase transition, i.e., some thermodynamic response functions possess a jump discontinuity. The inequality $\Theta>0$ defines a low density part of the phase space, connected smoothly to both phases at $\Theta<0$. Near the vapor-liquid critical point, the condition $\Theta>0>\Sigma$ approximately corresponds to the conventional definition of supercritical, which in an engineering context means that both pressure and temperature are above the pressure and temperature of the critical point. Critical points are characterized by $\Sigma=\Theta=0$. Generically, for fluid mixtures with $C$ components, they form a critical manifold of dimension $C-1$ (GRIfFITHS [24]) in the $(C+1)$-dimensional thermodynamic state space of the fluid.

The form of any EOS valid close to a critical point is strongly restricted by renormalization group arguments from statistical mechanics; see, e.g., Fisher [17], Zinn-Justin [63]. These give rise to various scaling laws for particular thermodynamic variables, as pressure, temperature, and chemical potential approach a critical point along specific trajectories, which must be reproduced by any accurate EOS.

The origin of the renormalization group and hence of the scaling laws is the fact that in a microscopic description of a macroscopic system, the mesoscopic length scale on which the thermal averaging is done can be changed without affecting the thermodynamic limit. In general, the renormalization group applies to an infinite number of scaling fields, of which only very few are relevant close to a critical point; their number equals the number of degrees of freedom near an isolated critical point. Since we can find isolated critical points in many mixtures at fixed composition, where thermodynamic states have only two degrees of freedom, there are only two ${ }^{4}$ relevant scaling fields $\Sigma$ and $\Theta$ in mixtures. The infinitely many remaining scaling correction fields $I_{1}, I_{2}, \ldots$ determine deviations from

\footnotetext{
${ }^{3}$ In traditional terminology, these are the nonlinear scaling fields. Close to the critical point, they can be approximated by linear scaling fields. For the lattice gas, the linear approximations of $\Sigma$, $\Theta$, and the ordering field $\Omega$ introduced later are the deviation of chemical potential, temperature, and density from the corresponding critical point data, while the dependent scaling field $D$ introduced later is approximately a linear combination of these and the deviation of the pressure from the critical point pressure.

More realistic fluids, and especially fluid mixtures, follow this pattern only roughly. Because of the lattice gas, the locus $\Sigma=0$ is sometimes called the critical isochore, and the locus $\Theta=0$ the critical isotherm, a misleading terminology when applied to fluid mixtures.

Less close to the critical point, additional nonlinear correction terms appear in all four scaling fields.

${ }^{4}$ If three or more near-critical phases coexist, there may be a nearby tricritical or multicritical point (Griffiths \& Widom [25], Hankey et al. [28], Griffiths [24], Mistura [43]), a situation not covered by the Ising universality class. An analysis of this situation requires further research; cf. footnote ${ }^{14}$ below.
} 
the power laws. ${ }^{5}$

The existence of the renormalization group implies ${ }^{6}$ that there is a scaling equation of state

$$
D(P, T, \mu)=S\left(\Sigma, \Theta, I_{1}, I_{2}, \ldots\right)
$$

with a three times continuously differentiable dependent scaling field $D=D(P, T, \mu)$ and an (except for $\Sigma=0>\Theta$ ) three times continuously differentiable scaling function $S$ satisfying the exact scaling relation

$$
\lambda S\left(\Sigma, \Theta, I_{1}, I_{2}, \ldots\right)=S\left(\lambda^{e_{-1}} \Sigma, \lambda^{e_{0}} \Theta, \lambda^{e_{1}} I_{1}, \lambda^{e_{2}} I_{2}, \ldots\right) \quad \text { for all } \lambda>0
$$

where

$$
1>e_{-1}>e_{0}>0>e_{1} \geq e_{2} \geq \ldots
$$

are universal (i.e., substance-independent) critical exponents. In a classical EOS, $D$, $\Theta$, and $\Sigma$ (or thermodynamic quantities derived from this, such as an order parameter) are related by an analytic nonlinear equation without any singularities. It is well-known that this leads to critical points with the same exponents as for the van der Waals EOS (in the present notation $e_{0}=\frac{1}{2}$ and $e_{-1}=\frac{3}{4}$ ), corresponding to the mean field approximation in statistical mechanics. These exponents match neither experimental data close to the critical point nor theoretical predictions from Ising-like models, whose universal features are valid for the whole Ising universality class. Accurate numerical values for the most important critical exponents ${ }^{7}$ are

$$
e_{-1}=0.82729(2), e_{0}=0.52908(9), e_{1}=-0.277(2), e_{2}=-0.56(3), e_{3}=-0.61(6)
$$

where the numbers in parentheses denote one standard deviation uncertainty per unit the last place. In particular, the EOS for every real fluid or fluid mixture is nonclassical and intrinsically nonanalytic near critical points.

All general universality and scaling properties are consequences of the scaling relation (2) for (1) and the known smoothness properties. In particular, both renormalization group

\footnotetext{
${ }^{5}$ Traditionally, the scaling correction fields were called irrelevant scaling fields, but at the presently available accuracies they are far from irrelevant numerically. The notation used in the literature for the scaling fields is not uniform. The notation used in Fisher [17] is related to the present one by $f=D$, $h_{1}=\Theta, h_{2}=\Sigma, h_{k+2}=I_{k}$. The notation used in Sengers \& Shanks [54] corresponds to $h_{1}=\Sigma$, $h_{2}=\Theta, h_{3}=D$.

${ }^{6}$ The traditional proofs guarantee the scaling law in the form of an asymptotic series only; so one expects the scaling law to hold at least close to the critical point. However, in the exactly solvable models of Fisher \& Felderhof $[18,19]$ and Reuter \& Bugaev [50], the scaling law can be seen to be valid globally.

${ }^{7}$ There is a multitude of related critical exponents for various critical scaling relations between particular thermodynamic observables; see, e.g., Pelissetto \& Vicari [46]. In dimension $d=3$, these are given with their traditional label by

$$
\alpha:=2-1 / e_{0}, \quad \beta:=\left(1-e_{-1}\right) / e_{0}, \quad \gamma:=\left(2 e_{-1}-1\right) / e_{0}, \quad \delta:=e_{-1} /\left(1-e_{-1}\right),
$$$$
\nu:=1 / 3 e_{0}, \quad \eta:=5-6 e_{-1}, \quad \omega:=-3 e_{1}, \quad y_{m}:=3 e_{m}, \quad \Delta_{m}:=-e_{m} / e_{0} .
$$

The currently best values, taken from Hasenbusch [29] and Newman \& Riedel [45, Table V] are

$$
\nu=0.63002(10), \quad \eta=0.03627(10), \quad \omega=0.832(6), \quad \Delta_{2}=0.98(6), \quad \Delta_{3}=1.07(11) .
$$

Using the propagation formulas $\sigma_{f(x)} \approx\left|f^{\prime}\left(\mu_{x}\right)\right| \sigma_{x}$ for the standard deviation $\sigma_{x}$ of a random variable $x$ with mean $\mu_{x}$, this yields the above values for $e_{-1}=(5-\eta) / 6, e_{0}=1 / 3 \nu, e_{1}=-\omega / 3$, and $e_{m}=y_{m} / 3$ $(m=2,3)$.
} 
theory and experimental evidence show that once the scaling fields are normalized to get rid of multipliers in their definition that do not affect the validity of the scaling relation, $S$ is a universal function of its arguments.

We now exploit special properties of the 3-dimensional Ising model. The first, well-known property is the fact that the Ising model has a reflection symmetry, which implies that the scaling function $S$ in (1) is an even function of $\Sigma$, hence depends on $\Sigma$ only through $\Sigma^{2}$,

$$
S\left(\Sigma, \Theta, I_{1}, I_{2}, \ldots\right)=s\left(\Sigma^{2}, \Theta, I_{1}, I_{2}, \ldots\right) .
$$

Since $S$ is a universal function, this holds generally in the Ising universality class. Thus, as far as fluids and fluid mixtures are described by the Ising universality class, they inherit this symmetry when the scaling fields are properly chosen.

The second property is a hitherto apparently unnoticed fact: For the 3-dimensional Ising model, and hence in all models from the 3-dimensional Ising universality class, the dependent scaling field $D$, which must vanish at the critical point, is positive in a punctured neighborhood of the critical point. This observation is crucial for the derivation of the new analytic representation of the critical EOS, as it allows us to make in (2) the special choice $\lambda:=D^{-1}$. We obtain

$$
S\left(D^{-e_{-1}} \Sigma, D^{-e_{0}} \Theta, D^{-e_{1}} I_{1}, D^{-e_{2}} I_{2}, \ldots\right)=D^{-1} S\left(\Sigma, \Theta, I_{1}, I_{2}, \ldots\right) \quad \text { for all } \lambda>0 .
$$

In view of (5), the EOS (1) takes the implicit form

$$
s\left(D^{-2 e_{-1}} \Sigma^{2}, D^{-e_{0}} \Theta, D^{-e_{1}} I_{1}, D^{-e_{2}} I_{2}, \ldots\right)=1 .
$$

Remarkably, the thermodynamic state space defined by an implicit equation of this form is, independent of the particular choice of the function $s$, automatically invariant under the scaling transformation

$$
\Sigma \rightarrow \lambda^{e_{-1}} \Sigma, \quad \Theta \rightarrow \lambda^{e_{0}} \Theta, \quad D \rightarrow \lambda D, \quad I_{k} \rightarrow \lambda^{e_{k}} I_{k}
$$

with arbitrary $\lambda>0$. Hence (6) is a full embodiment of all universality and critical scaling properties, even when $s$ itself satisfies no scaling relation. Of course, the function $s$ must be such that, at least near a critical point, ome may solve (6) for $D$ when $\Sigma, \Theta, I_{1}, I_{2}, \ldots$ are given. Should there be more than one solution, thermodynamic stability considerations ${ }^{8}$ imply that the largest solution describes the stable phase. In case of ties, several phases coexist; cf. Neumaier [44].

Very close to the critical point, the scaling correction fields can be neglected, and (1) simplifies to

$$
D=S^{\mathrm{crit}}(\Sigma, \Theta)
$$

where

$$
S^{\mathrm{crit}}(\Sigma, \Theta):=S(\Sigma, \Theta, 0,0, \ldots) .
$$

${ }^{8}$ For two phases of a pure substance, $D, \Theta$, and $\Sigma$ are the renormalized analogue of pressure, temperature, and molar Gibbs free energy (which for pure substances equals the chemical potential). Therefore the stable phase at fixed $\Theta$ consists of the branches with the smallest value of $\Sigma$ at fixed $D$. Drawing a $(D, \Sigma)$ diagram then shows that $D$ has the largest value at fixed $\Sigma$. 
Similarly, (6) simplifies to

$$
s^{\mathrm{crit}}\left(D^{-2 e_{-1}} \Sigma^{2}, D^{-e_{0}} \Theta\right)=1,
$$

where $s^{\text {crit }}$ is an analytic function but need not satisfy a scaling relation.

However, unless both $\Sigma$ and $\Theta$ are tiny, the influence of (at least) the first scaling correction field is significant and leads to so-called corrections to scaling (WEGNER [59]).

\section{Order parameter and generalized polar coordinates}

Traditionally, the properties close to the critical point (where all scaling correction fields $I_{k}$ may be neglected) are discussed in terms of a thermodynamic relations between $|\Sigma|, \Theta$, and the asymptotic order parameter ${ }^{9}$

$$
\Omega:=\left(\frac{d D}{d \Sigma}\right)_{\Theta}=\frac{d}{d \Sigma} S^{\mathrm{crit}}(\Sigma, \Theta) .
$$

Following ScHOFIELD [52], this is done by a parameterization in terms of variables adapted to the geometry near a critical point, chosen to remove the singularity at the critical point. In a parametric representation, the relevant fields are expressed in terms of a radial parameter $r \geq 0$, a measure of distance from the critical point, and an angular parameter $\varphi \in\left[-\varphi_{\max }, \varphi_{\max }\right]$, taking the values $\varphi= \pm \varphi_{\max }$ at the two sides of the coexistence curve. Close to the critical point, asymptotic scaling properties hold for any parameterization in terms of generalized polar coordinates of the form

$$
D=r^{e_{D}} c_{D}\left(\varphi^{2}\right), \quad \Sigma=r^{e_{\Sigma}} \varphi c_{\Sigma}\left(\varphi^{2}\right), \quad \Theta=r^{e_{\Theta}} c_{\Theta}\left(\varphi^{2}\right), \quad \Omega=r^{e_{\Omega}} \varphi c_{\Omega}\left(\varphi^{2}\right)
$$

for $r \geq 0$ and $|\varphi| \leq \varphi_{\max }$, with appropriate critical exponents $e_{D}, e_{\Theta}, e_{\Sigma}, e_{\Omega}$ and analytic functions $c_{D} \geq 0, c_{\Theta}, c_{\Sigma} \geq 0$, and $c_{\Omega} \geq 0$ of the single variable

$$
\xi:=\varphi^{2} \in\left[0, \xi_{\max }\right], \quad \xi_{\max }:=\varphi_{\max }^{2} .
$$

The particular form (12) of the parameterization is chosen to reflect the reflection symmetry of the Ising model and the fact that for $\Sigma \rightarrow \pm 0$, the order parameter $\Omega$ vanishes if $\Theta \geq 0$, whereas for $\Theta<0$, it has the sign of $\Sigma$ and grows with the distance from the critical point.

\footnotetext{
${ }^{9}$ For the statistical mechanics treatment of the Ising model, the appropriate language uses a "magnetic" terminology. There, in the immediate neighborhood of the critical point, the thermal scaling field $\Theta$ is proportional to $T-T^{\text {crit }}$, where $T^{\text {crit }}$ is the temperature at the critical point, the strong scaling field $\Sigma$ is proportional to the magnetic field $H$, the order parameter $\Omega$ is proportional to the magnetization $M$, and the dependent scaling field $D$ is proportional to the Gibbs free energy $V^{-1} \log Z$ minus an analytic function of $T$. (Much of the discussion in the literature simply equates $\Theta=T-T^{\text {crit }}, \Sigma=H$, and $\Omega=M$.)

The proportionality factors are the only nonuniversal features in the relations between $\Sigma, \Theta, \Omega$, and $D$. Further away from the critical point, additional nonlinear terms are needed: Thus $D$ is $V^{-1} \log Z$ minus a function of $T-T_{c}$ and $H$, even in $H, \Theta$ is a function of $T-T_{c}$ and $H$, even in $H$, and $\Sigma$ is a function of $T-T_{c}$ and $H$, odd in $H$. In this case, one must also account for the scaling correction fields.
} 
In order to be equivalent to (8), the parameterizing functions $c_{D}, c_{\Theta}, c_{\Sigma}$, and $c_{\Omega}$ must, in addition to the above sign constraints, satisfy some simple conditions that guarantee unique coordinates for the $(\Theta, \Sigma)$-plane: For $\xi \in\left[0, \xi_{\max }\right], c_{\Sigma}$ must vanish at $\xi=\xi_{\max }$, and $c_{\Theta}$ must vanish at $\xi=\xi_{0}$ for some $\xi_{0}$ with $0<\xi_{0}<\xi_{\max }$. Moreover, $|\Sigma|^{-e_{\Theta} / e_{\Sigma}} \Theta$ must take every real value just once. This requires that

$$
Y(\xi):=\frac{c_{\Theta}(\xi)}{\left(\sqrt{\xi} c_{\Sigma}(\xi)\right)^{e_{\Theta} / e_{\Sigma}}}
$$

the analogue of the cotangent function for ordinary polar coordinates, must be strictly monotone decreasing.

The invariance under the scaling relations (7) is ensured for the critical exponents

$$
e_{D}=1, \quad e_{\Sigma}=e_{-1}, \quad e_{\Theta}=e_{0}, \quad e_{\Omega}=1-e_{-1}
$$

But the critical exponents are determined only up to a common factor, as the reparameterization $r^{\prime}=r^{k}$ shows. Their ratios are universal and determine the traditional critical exponents

$$
\begin{gathered}
\beta:=\frac{e_{\Omega}}{e_{\Theta}}=\frac{1-e_{-1}}{e_{0}}, \quad \delta:=\frac{e_{\Sigma}}{e_{\Omega}}=\frac{e_{-1}}{1-e_{-1}}, \\
2-\alpha:=\frac{e_{D}}{e_{\Theta}}=\frac{1}{e_{0}}=\beta(\delta+1)
\end{gathered}
$$

in terms of which

$$
e_{-1}=\frac{\delta}{\delta+1}, \quad e_{0}=\frac{1}{2-\alpha}=\frac{1}{\beta(\delta+1)}
$$

Without loss of generality, the free factor may be chosen to make $e_{\Theta}=1$. If this choice is made, the most general setting given above reduces to the situation

$$
e_{D}=2-\alpha=\beta(\delta+1), \quad e_{\Sigma}=\beta \delta, \quad e_{\Theta}=1, \quad e_{\Omega}=\beta,
$$

extensively discussed in Pelissetto \& ViCARI [46]. The relations are spelled out in more details in Appendix A.

At fixed $\Theta \neq 0$, we have for all $\varphi>0$

$$
\begin{gathered}
r=\left(\frac{\Theta}{c_{\Theta}\left(\varphi^{2}\right)}\right)^{1 / e_{\Theta}}, \\
D=|\Theta|^{\beta(\delta+1)} \frac{c_{D}\left(\varphi^{2}\right)}{\left|c_{\Theta}\left(\varphi^{2}\right)\right|^{\beta(\delta+1)}}, \quad \Sigma=|\Theta|^{\beta \delta} \frac{\varphi c_{\Sigma}\left(\varphi^{2}\right)}{\left|c_{\Theta}\left(\varphi^{2}\right)\right|^{\beta \delta}},
\end{gathered}
$$

hence (writing $\left.c^{\prime}(\xi):=d c(\xi) / d \xi\right)$

$$
\begin{aligned}
\left(\frac{d D}{d \varphi}\right)_{\Theta} & =|\Theta|^{\beta(\delta+1)} \frac{c_{D}^{\prime} 2 \varphi\left|c_{\Theta}\right|^{\beta(\delta+1)}-c_{D} \beta(\delta+1)\left|c_{\Theta}\right|^{\beta(\delta+1)-1}\left|c_{\Theta}\right|^{\prime} 2 \varphi}{\left|c_{\Theta}\right|^{2 \beta(\delta+1)}} \\
& =|\Theta|^{\beta(\delta+1)} \frac{2 \varphi\left(c_{D}^{\prime}\left|c_{\Theta}\right|-\beta(\delta+1) c_{D}\left|c_{\Theta}\right|^{\prime}\right)}{\left|c_{\Theta}\right|^{\beta(\delta+1)+1}}
\end{aligned}
$$




$$
\begin{aligned}
\left(\frac{d \Sigma}{d \varphi}\right)_{\Theta} & =|\Theta|^{\beta \delta} \frac{\left(c_{\Sigma}+\varphi c_{\Sigma}^{\prime} 2 \varphi\right)\left|c_{\Theta}\right|^{\beta \delta}-\varphi c_{\Sigma} \beta \delta\left|c_{\Theta}\right|^{\beta \delta-1}\left|c_{\Theta}\right|^{\prime} 2 \varphi}{\left|c_{\Theta}\right|^{2 \beta \delta}} \\
& =|\Theta|^{\beta \delta} \frac{c_{\Sigma}\left|c_{\Theta}\right|+2 \varphi^{2}\left(c_{\Sigma}^{\prime}\left|c_{\Theta}\right|-\beta \delta c_{\Sigma}\left|c_{\Theta}\right|^{\prime}\right)}{\left|c_{\Theta}\right|^{\beta \delta+1}} .
\end{aligned}
$$

Therefore

$$
\left(\frac{d D}{d \Sigma}\right)_{\Theta}=|\Theta|^{\beta} \frac{\varphi c_{\Omega}\left(\varphi^{2}\right)}{\left|c_{\Theta}\right|\left(\varphi^{2}\right)^{\beta}}=\Omega
$$

where

$$
c_{\Omega}=\frac{c_{D}^{\prime}\left|c_{\Theta}\right|-\beta(\delta+1) c_{D}\left|c_{\Theta}\right|^{\prime}}{\frac{1}{2} c_{\Sigma}\left|c_{\Theta}\right|+\xi\left(c_{\Sigma}^{\prime}\left|c_{\Theta}\right|-\beta \delta c_{\Sigma}\left|c_{\Theta}\right|^{\prime}\right)}=\frac{c_{D}^{\prime} c_{\Theta}-\beta(\delta+1) c_{D} c_{\Theta}^{\prime}}{\frac{1}{2} c_{\Sigma} c_{\Theta}+\xi\left(c_{\Sigma}^{\prime} c_{\Theta}-\beta \delta c_{\Sigma} c_{\Theta}^{\prime}\right)} .
$$

By reflection symmetry, the same result also holds for $\varphi<0$, and hence generally.

The parameterizing functions $c_{D}, c_{\Theta}, c_{\Sigma}$, and $c_{\Omega}$ determine the precise form of the implicit dependence of $D, \Theta, \Sigma$, and $\Omega$ defined by (12). Two of the parameterizing functions may be chosen fairly freely and determine the coordinate transformation; the remaining ones are then fixed by the relation (18) and the universal EOS. The initially unknown universal part is determined numerically from fits to data from experiment or from perturbative microscopic calculations or statistical simulation of a statistical mechanics model from the Ising universality class.

\section{A $\Sigma$-explicit critical EOS}

Continuing the discussion of Section 3, we now discuss in more details numerical results for a special choice of generalized polar coordinates. We use the critical exponents (14) and define coordinates through

$$
c_{D}(\xi)=1, \quad c_{\Sigma}(\xi)=C\left(\theta_{\max }-\xi\right), \quad c_{\Theta}(\xi)=\theta_{\max }-\xi, \quad c_{\Omega}(\xi)=B\left(\theta_{\max }-\xi\right)^{-1}
$$

with analytic functions $C(\theta)=C^{\text {crit }}(\theta)$ and $B(\theta)=B^{\text {crit }}(\theta)$ of

$$
\theta:=\theta_{\max }-\xi .
$$

From (12), (14), and (19), we get the explicit coordinate definitions

$$
r:=D, \quad \theta:=D^{-e_{0}} \Theta, \quad \varphi:=\operatorname{sign} \Sigma \sqrt{\theta_{\max }-\theta}
$$

and the asymptotic scaling EOS

$$
\Sigma^{2}=D^{2 e_{-1}} W^{\text {crit }}\left(D^{-e_{0}} \Theta\right), \quad \Omega^{2}=D^{2\left(1-e_{-1}\right)} \Delta^{\text {crit }}\left(D^{-e_{0}} \Theta\right), \quad \operatorname{sign} \Omega=\operatorname{sign} \Sigma,
$$

with analytic

$$
W^{\text {crit }}(\theta)=\left(\theta_{\max }-\theta\right) C^{\text {crit }}(\theta)^{2}, \quad \Delta^{\text {crit }}(\theta)=\left(\theta_{\max }-\theta\right) B^{\text {crit }}(\theta)^{-2} .
$$

(21) captures the asymptotic critical behavior in a simple and comprehensive way. (21) is explicit in $\Sigma^{2}$, and has the form (10) with

$$
s^{\mathrm{crit}}(\sigma, \theta)=1+\sigma^{2}-W^{\mathrm{crit}}(\theta), \quad \sigma:=D^{-e_{-1}} \Sigma .
$$


In particular, $W^{\text {crit }}$ can be obtained from the scaling function $S$ in (5) by solving (1) for $\Sigma^{2}$. This proves that $W^{\text {crit }}$ has the same differentiability properties as the scaling function $S$. In the literature, this scaling function is generally assumed to be analytic except for a possible singularity at $\Theta=0$. This is based on arguments of GRIfFiths [23] who suggested what is now called Griffiths analyticity, that thermodynamic functions should be analytic except at the critical point. We show in Appendix A that assuming $W^{\text {crit }}$ to be analytic has the same consequences as Griffiths analyticity for the high-temperature and low temperature expansions that fully capture the critical behavior.

The parameter $\theta$ takes values only in a finite interval $\left[\theta_{\text {coex }}, \theta_{\max }\right]$, where

$$
\theta_{\text {coex }}:=\theta_{\max }-\xi_{\max }<0
$$

and we have $\theta=\theta_{\text {coex }}$ along the coexistence manifold. The asymptotic universal function $W^{\text {crit }}(\theta)$ is positive for $\theta_{\text {coex }}<\theta<\theta_{\max }$ and vanishes at both end points, with a double zero at $\theta=\theta_{\text {coex }}$. Thus we have (22) with a real analytic, nonnegative function $C^{\text {crit }}$ of $\theta \in\left[\theta_{\text {coex }}, \theta_{\max }\right]$. We may normalize the thermal scaling field such that $\theta_{\text {coex }}=-1$; then $\theta_{\max }$ is a universal constant, and we may normalize the strong scaling field such that

$$
C^{\text {crit }}(-1)=0, \quad C^{\text {crit }}(0.5)=1.5 \text {. }
$$

The normalization by $C^{\text {crit }}(0.5)=1.5$ is a convenient choice satisfied for

$$
C_{\text {lin }}^{\text {crit }}(\theta)=1+\theta
$$

which (cf. Figure 1) already gives a reasonable first approximation $W_{\text {lin }}^{\text {crit }}(\theta)$ to $W^{\text {crit }}(\theta)$.

Since $\Sigma=0$ is equivalent to $W^{\text {crit }}(\theta)=0$ and $\Theta<0$ on the coexistence manifold, we must have there $C^{\operatorname{crit}}(\theta)=0$, hence $\theta=-1$. Therefore the equation for the coexistence manifold simply becomes

$$
\Theta(P, T, \mu)=-D(P, T, \mu)^{e_{0}}
$$

From (18), (19), and (16) we deduce the formula

$$
B(\theta)=\frac{e_{0} \theta}{2} C(\theta)+\left(\theta_{\max }-\theta\right)\left(e_{-1} C(\theta)-e_{0} \theta C^{\prime}(\theta)\right)
$$

With the normalization (23), $C(\theta)$ and hence $W^{\text {crit }}(\theta)$ and $B(\theta)$ are universal analytic functions of $\theta \in\left[-1, \theta_{\max }\right]$, and we have $C(\theta)>0$ for $-1<\theta<\theta_{\max }$.

The detailed information about the form of the universal function $W^{\text {crit }}$ just spelled out may be derived from computations based on microscopic models of a fluid. Using information from Butera \& Pernici [10] (see Appendix B for more details) we find the approximation

$$
\begin{gathered}
C^{\text {crit }}(\theta)=(\theta+1)(1-(\theta-0.5) c(\theta)) \\
c(\theta)=0.03339+0.005356 \theta+0.001185 \theta^{2}, \quad \theta_{\max }=1.39444 .
\end{gathered}
$$

Figure 1 displays $W^{\text {crit }}, C^{\text {crit }}(\theta), B^{\text {crit }}(\theta)$ for this choice and for the simpler approximation (24). 
Figure 1: The asymptotic universal functions $C(\theta), B(\theta)$, and $W(\theta)$, and the "linear" approximation (dashed) from (24).
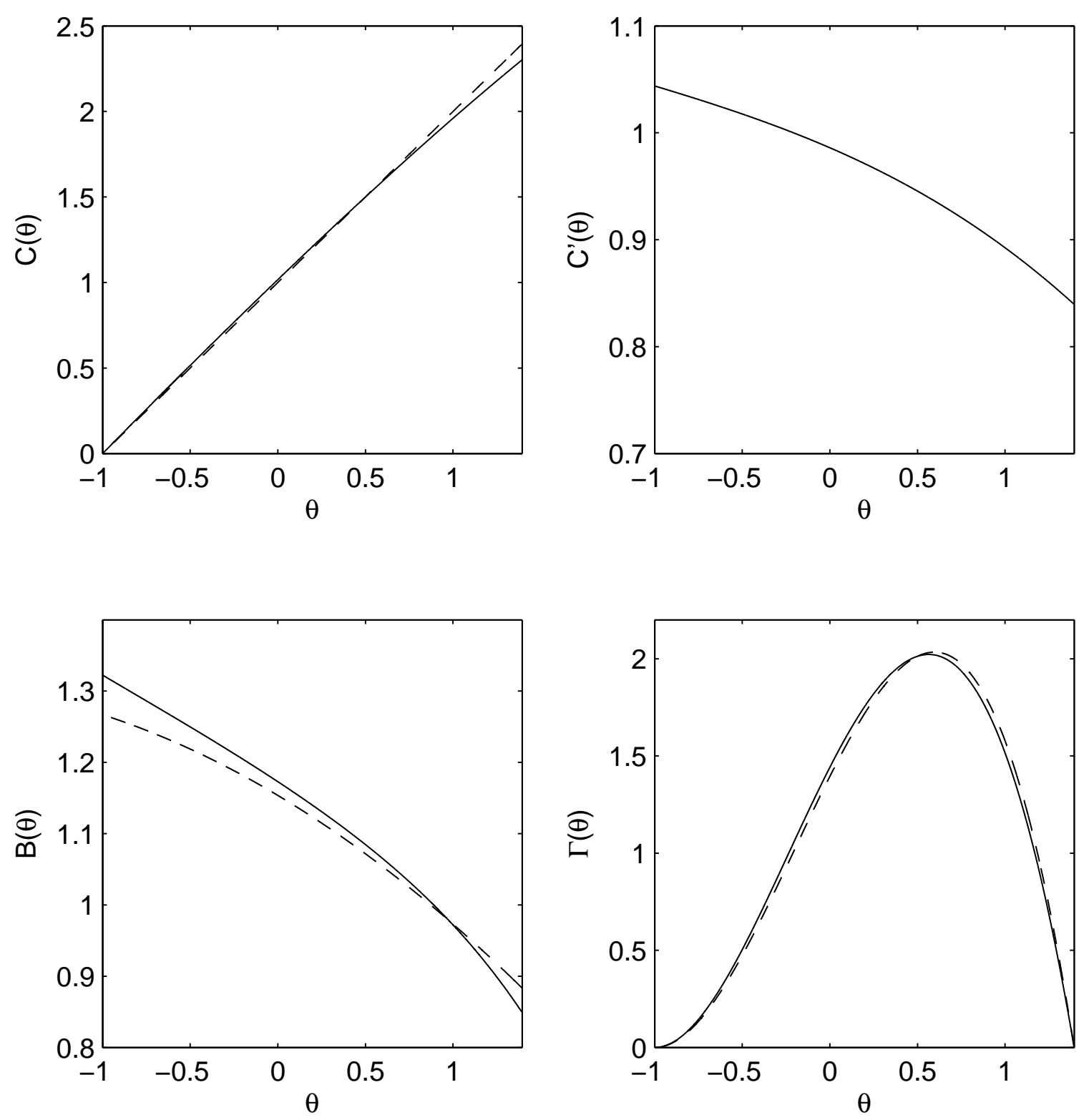

\section{A $D$-explicit formulation}

Although (21) is a complete and fully adequate description of the thermodynamic state space close to the critical point, we now discuss whether we can also find a smooth representation of the $D$-explicit form (8). There is a generally accepted belief that it " $i s$ not possible to write the scaled expression for $D$ as an explicit function of $\Sigma$ and $\Theta$. Such attempts always cause singular behavior of the thermodynamic potential in the one-phase region either at $\Sigma=0$ or at $\Theta=0$." (This quotes a passage after equation (20) of the 
Figure 2: The asymptotic universal function $r(Q)$ for $a=1$.

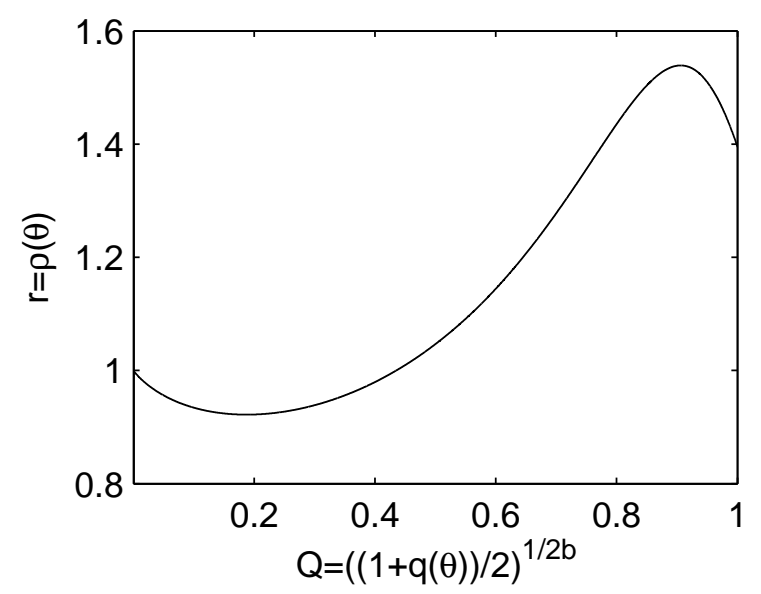

recent paper by HoLTEN et al. [31], adapted to the present notation. Essentially the same statement can be found in BehneJAD et al. [6, Section 10.2.2]. Related issues are discussed in Gaunt \& Domb [22] and Vicentini-Missoni [57, Section IV].)

The results of the previous section allow us to clarify the extent to which this belief is justified, exposing hidden assumptions on which it is based. To do this, we choose an arbitrary $a>0$ and define

$$
\begin{gathered}
b:=\frac{e_{0}}{e_{-1}}=\frac{1}{\beta \delta} \approx 0.63953, \\
\rho(\theta):=\sqrt{\theta^{2}+a W^{\mathrm{crit}}(\theta)^{b}}, \\
q(\theta):=\frac{\theta}{\rho(\theta)} .
\end{gathered}
$$

Clearly, $|q(\theta)| \leq 1$, so

$$
Q(\theta):=\left(\frac{1+q(\theta)}{2}\right)^{1 / 2 b} \in[0,1]
$$

Figure 2 shows that

$$
\rho(\theta)=r(Q(\theta))
$$

with a function $r(Q)$ that, by construction, is analytic for $Q \in] 0,1[$. At the boundary, $r(Q)$ is continuously differentiable but has higher order singularities, with

$$
r(\delta)=1-c \delta+O\left(\delta^{2 b}\right), \quad r(1-\delta)=\theta_{\max }+c^{\prime} \delta+O\left(\delta^{1 / b}\right) \quad \text { for } \delta \downarrow 0 .
$$

This follows since with appropriate positive constants $c_{i}$, we have for $\theta=-1+\varepsilon$ and $\varepsilon \downarrow 0$,

$$
W^{\mathrm{crit}}(\theta)=c_{1} \varepsilon^{2}+O\left(\varepsilon^{2}\right), \quad \rho=1-\varepsilon+O\left(\varepsilon^{2 b}\right), \quad q=-1+O\left(\varepsilon^{2 b}\right), \quad Q=O(\varepsilon),
$$

and for $\theta=\theta_{\max }-\varepsilon$ and $\varepsilon \downarrow 0$,

$W^{\text {crit }}(\theta)=c_{2} \varepsilon+O\left(\varepsilon^{2}\right), \quad \rho=\theta_{\max }+c_{3} \varepsilon^{b}-\varepsilon+O\left(\varepsilon^{1+b}\right), \quad q=1-O\left(\varepsilon^{b}\right), \quad Q=1-O\left(\varepsilon^{b}\right)$. 
Using (20) and (21), we find

$$
\begin{gathered}
R:=\sqrt{\Theta^{2}+a|\Sigma|^{2 b}}=D^{e_{0}} \rho(\theta), \\
\Theta=R q(\theta),
\end{gathered}
$$

so that in view of $(28)$,

$$
D=S^{\mathrm{crit}}(\Sigma, \Theta):=\left(\frac{R}{r(\Theta / R)}\right)^{1 / e_{0}} .
$$

Thus we have represented $D$ explicitly as a function of $\Theta$ and $\Sigma$, as in (8).

By construction, $S^{\text {crit }}(\Sigma, \Theta)$ is analytic except possibly at $\Theta / R \in\{0,1\}$, which corresponds to $\Sigma=0$. However, a similar argument shows that the formula (29) also holds with

$$
\widetilde{\rho}(\theta):=\left(W^{\mathrm{crit}}(\theta)+a|\theta|^{c}\right)^{1 / c}, \quad \widetilde{R}:=\left(\Sigma^{2}+a|\Theta|^{c}\right)^{1 / c}
$$

in place of $R$ and $\rho(\theta)$, where $c:=2 e_{-1} / e_{0} \approx 3.12728$. The resulting alternative expression shows that $S^{\text {crit }}(\Sigma, \Theta)$ (which must obviously be independent of the way it is represented) is analytic except possibly at $\Theta$. We conclude that $S^{\text {crit }}(\Sigma, \Theta)$ is in fact analytic throughout the one-phase region. ${ }^{10}$

However, when $r(Q)$ is approximated by a polynomial or another analytic function, it has no longer the correct nonanalytic structure at the boundary to cancel the nonanalyticity in $R$, and the resulting approximation to $S^{\text {crit }}(\Sigma, \Theta)$ becomes nonanalytic at $\Sigma=0$.

As (29) shows, it is possible to write $D$ as an analytic and explicit function of $\Sigma$ and $\Theta$. In this formulation, the lack of analyticity in the one-phase region alluded to in the statement quoted above appears only when one approximates the exact $r(Q)$ by a polynomial, and can therefore, in principle, be made numerically arbitrarily small. But since a highly accurate approximation of nonanalytic functions by polynomials needs a very high polynomial degree, we do not recommend the $D$-explicit form for numerical work.

The $\Sigma$-explicit EOS does not share these approximation difficulties, since $C(\theta)$ is analytic in the closed interval $\left[-1, \theta_{\max }\right]$ and easy to approximate by low degree polynomials.

\section{A global $\Sigma$-explicit scaling equation of state}

While it is unclear how to extend Schofield's generalized polar coordinates approach to the case further away from the critical point, when the scaling corrections cannot be neglected, the $\Sigma$-explicit form has an immediate generalization.

\footnotetext{
${ }^{10}$ Thus the nonanalyticity in $R$ is completely cancelled by that in $r(Q)$. Such a complete cancellation is not necessarily the case for other critical systems. For example, the simplified but exactly solvable 1-dimensional model of a fluid with a critical point discussed by FisHer \& FELDERHOF [18, 19] - whose $\Sigma$-explicit EOS, given in footnote ${ }^{11}$, is slightly different from that discussed here - , has a continuous phase transition for $\Theta=0>\Sigma$, corresponding to nonanalytic behavior of $S^{\operatorname{crit}}(\Sigma, \Theta)$ at this locus. (They call $\Theta=0$ the limit locus and $\Sigma=0$ the vapor-pressure curve.)
} 
The new formulation provides us with very useful additional flexibility. Indeed, if we rearrange the equation (6) into any algebraically equivalent form, the content of the equation remains unchanged. This means that many different functions $s$ express the same constraint on the thermodynamic state space, and we can look for a normal form that is simpler and restores the uniqueness. It turns out that for states close to a critical point belonging to the Ising universality class, we can solve (6) uniquely for $\Sigma^{2}$. This ${ }^{11}$ allows us to cast the equation as a scaling EOS in normal form

$$
\Sigma^{2}=D^{2 e_{-1}} W\left(D^{-e_{0}} \Theta, D^{-e_{1}} I_{1}, D^{-e_{2}} I_{2}, \ldots\right), \quad D>0,
$$

valid except at critical points, where $D=\Sigma=\Theta=0$. Note that (30) is explicit in $\Sigma^{2}$ rather than in $D$. The squared scaling field reflects the mirror symmetry of the Ising model and allows for phase transitions at $\Sigma=0$.

Generalizing from the asymptotic situation close to a critical point, discussed above, the scaling EOS holds for the Ising universality class in the $\Sigma$-explicit normal form (30) with

$$
W\left(\theta, \iota_{1}, \iota_{2}, \ldots\right)=\left(L\left(\iota_{1}, \iota_{2}, \ldots\right)-\theta\right) C\left(\theta, \iota_{1}, \iota_{2}, \ldots\right)^{2},
$$

with universal nonnegative, analytic functions $L$ and $C$ satisfying

$$
L(0,0, \ldots)=\theta_{\max }, \quad C(\theta, 0,0, \ldots)=C^{\text {crit }}(\theta) .
$$

As a consequence, the coexistence manifold $\Sigma=0>\Theta$ is now characterized by the equation $C\left(\theta, \iota_{1}, \iota_{2}, \ldots\right)=0$. The equation for the coexistence manifold therefore takes the form

$$
C\left(D^{-e_{0}} \Theta, D^{-e_{1}} I_{1}, D^{-e_{2}} I_{2}, \ldots\right)=0 .
$$

The novelty is that, in this form, $W$ is a universal analytic function of its arguments. Since $D>0$ off the critical manifold, the powers of $D$ appearing in (30) introduce no singularities in the thermodynamic state space. Thus away from the critical manifold, $\Sigma^{2}$ is also an analytic function of $D, \Theta$, and the $I_{k}$. The nonanalyticity shows only upon trying to solve for $D$ (or variables on which $D$ depends).

The derivation of (30) and (31) is valid only close to a critical point, hence (30) is guaranteed to hold only there. However, if $B$ and $C$ are analytic functions without singularities (an assumption similar to Griffiths analyticity; cf. GRIFFITHS [23]) then analytic continuation implies its global validity.

It would be interesting to find explicit numerical expressions for the universal functions $L$ and $C$. However, only limited information is available at present about the numerical details of the corrections to scaling; see, e.g., Butera \& Comi [9], CAmpostrini et al. [13], Hasenbusch [29], Zhong \& Barmatz [62].

\footnotetext{
11 The exactly solvable model fluid of Fisher \& Felderhof [19] possesses close to the critical point an asymptotic scaling EOS of the closely related form $\Sigma=D^{e_{-1}} X\left(D^{-e_{0}} \Theta\right)$. The variables of their case (Aii) are related to the present notation by

$$
\xi=\Sigma, \quad \theta=\Theta, \quad \omega=D, \quad T_{c}=1+e_{-1}, \quad \sigma=e_{0} .
$$
}




\section{$7 \quad$ Phenomenological scaling models for fluid mixtures}

To apply the general results to specific fluid mixtures, one needs to take the substancespecific information into account. It enters through the way the scaling fields $\Theta=\Theta(T, \mu)$, $\Sigma=\Sigma(T, \mu), I_{k}=I_{k}(T, \mu)$, and $D=D(P, T, \mu)$ are expressed in terms of the basic thermodynamic variables. Apart from the smoothness requirement, the general theory is silent about their form, which varies from system to system. The literature contains a large variety of phenomenological scaling models that provide reasonable formulas for the EOS of fluid mixtures that can be related to experimental information.

In the first paper modeling (binary) mixtures with correct scaling properties close to the critical point, LEUNG \& GRIFfiths [41] express everything in terms of force field variables. For industrial applications, multicomponent formulations in a cubic EOS, Helmholtz or Gibbs free energy framework are desirable, so that the composition can be kept constant. A number of such formulations were presented in the literature; see, e.g., $[2,7,15,32,35,36,37,48]$. However, formulations at fixed composition cannot match exactly the singularities; indeed WHEELER \& GRIFFITHS [61] prove that when some mole fractions are held constant, curves of plait points have bounded heat capacity, while the heat capacity at the critical point of a pure substance diverges. A more detailed analysis leads to additional renormalization phenomena (FISHER [16]). Ignoring these, as often done in these formulations, therefore requires additional approximations which may result in artifacts very close to the critical point (KISELEV \& FRIEND [36]). Other papers (e.g., $[11,12])$ implement the renormalization group approach more directly, resulting in an iterative definition of an equation of state that in the limit of infinitely many iterations satisfies the correct scaling laws. A thorough discussion of many practically relevant issues is given in the surveys by Anisimov \& Sengers [3] and Behnejad et al. [6].

In most models for critical fluids, $D$ is represented in the special form

$$
D(P, T, \mu)=P_{\text {reg }}(T, \mu)-P
$$

with a smooth function $P_{\text {reg }}$ of $T$ and $\mu$, and the remaining scaling fields $\Sigma, \Theta$ and the $I_{k}$ are independent of $P$. One characterizes this situation by saying that the resulting scaling laws are based on the assumption of revised scaling (REHR \& MERMIN [49]). Under these assumptions, (1) gives an EOS expressing the pressure ${ }^{12}$ explicitly as a function of temperature and chemical potential,

$$
P=P_{\text {reg }}(T, \mu)-\Delta(T, \mu),
$$

where

$$
\Delta(T, \mu):=S\left(\Sigma(T, \mu), \Theta(T, \mu), I_{1}(T, \mu), I_{2}(T, \mu), \ldots\right)
$$

is interpreted as a singular crossover term correcting the classical mean field contribution $P_{\text {reg }}(T, \mu)$ to the pressure in order to ensure the correct critical behavior.

However, the analysis of recent experiments suggests that revised scaling is not sufficient for some fluids; see the discussion in BERTRAND et al. [8]. Revised scaling also appears

\footnotetext{
${ }^{12}$ In "magnetic" language, the pressure is essentially the free energy.
} 
to be not general enough for theoretical reasons; e.g., the scaling fields of the model fluid of FisHER \& FELDERHOF [19] respect all standard requirements of statistical mechanics but do not satisfy the assumption of revised scaling. The more general complete scaling approach developed by Fisher \& OrkOUlAs [20, 33], although without a strong theoretical foundation, ${ }^{13}$ covers the above exceptions from revised scaling. In this approach, $\Theta$ and $\Sigma$ are allowed to be pressure-dependent, so that pressure, temperature and chemical potential enter symmetrically into the relevant scaling fields. In particular, BERTRAND et al. [8] suggest that current experimental critical data are consistent with keeping $\Theta$ pressure-independent, while $\Sigma$ must slightly depend on $P$ to correctly account for the so-called Yang-Yang anomaly [20].

Note that once $\Sigma$ or $\Theta$ is $P$-dependent, there is no particular reason why one should abolish the asymmetry in pressure, temperature and chemical potential while keeping an asymmetry in the scaling fields by restricting $D$ to the special form (33). Thus we propose to allow $D(P, T, \mu)$ to have an arbitrary smooth $P$-dependence. This leads to a more complete symmetry between the scaling fields and more flexibility in the adaptation to experimental data.

\section{A multiphase critical equation of state}

Most previous critical EOS are limited in several different ways:

- Frequently, the thermal scaling field $\Theta$ is taken to be linear in the temperature. However, linearity in $T$ limits the EOS to a narrow range of temperatures. Scaling fields with a more favorable temperature dependence such as one linear in $T^{-1}$ or $\tanh \left(T_{0} / T\right)$ extrapolate much better to the high temperature regime; see, e.g., Lundow \& CAMPBELL [42].

- Almost all studies attempting to go beyond the immediate neighborhood of the critical point work in the simplified setting of revised scaling, which does not account for all observable fluid behavior. The only previous EOS not restricted to revised scaling is the crossover EOS of BAKHSHANDEH \& BEHNEJAD [4, 5], which employs complete scaling, with scaling fields linear in $P, T$, and $\mu$.

- All noniterative equations of state with correct critical scaling are currently based on an implicit representation in terms of a Schofield type parameterization.

- Most papers discuss the two-phase case only. The only exception is RAINwATER [47], who attempts to cover vapor-liquid-liquid equilibrium. The main reason for this lack of generality seems to be that (as Rainwater's paper shows) a crossover mechanism in terms of a Schofield type parameterization is very difficult to extend to the multiphase case, since the Schofield parameters have no clear meaning far from the critical point and tend to introduce unphysical artifacts such as two coexisting vapor phases.

\footnotetext{
${ }^{13}$ It seems that all current theoretical derivations of the scaling laws (1)-(2) from renormalization group arguments apply to fluids only in the context of revised scaling. A derivation that provides a theoretical justification for complete scaling would be welcome.
} 
To construct an EOS suitable for the description of multiple phases even close to a critical point, we partition the phases into groups $g$ completely separated by a phase space boundary, while the phases within each group may be connected with each other by paths in phase space not crossing the coexistence manifold. One of these groups consists of all fluid phases; for solid phases, the groups may consist of a single phase only or (as, e.g., for $\beta$-brass, cf. LAMERS \& SchweIKA [39]) may contain several phases related by a critical point. The phases within each group are described by common, substance-specific scaling fields $\Sigma_{g}(P, T, \mu), \Theta_{g}(P, T, \mu)$, and $D_{g}(P, T, \mu)$, one for each phase group $g$. According to the results in Section 6, the critical behavior ${ }^{14}$ is correctly modelled if the EOS for each phase group takes the form

$$
s\left(D_{g}^{-2 e_{-1}} \Sigma_{g}^{2}, D_{g}^{-e_{0}} \Theta_{g}, D_{g}^{-e_{1}} I_{g 1}, D_{g}^{-e_{2}} I_{g 2}, \ldots\right)=1
$$

with a universal, substance-independent function $s$.

(34) is a nonlinear equation repating $P, T$, and $\mu$. Standard thermodynamic stability considerations imply (cf. Neumaier [44]) that, for given $T$ and $\mu$, the stable phase is determined by finding all solutions $P$ of the equations (34) for all phase groups $g$, and taking the one with largest $P$. In case of ties, several phases from different phase groups coexist. The Gibbs phase rule for the maximal number of coexistent phases is an automatic con sequence of this stability rule.

In particular, (34) is the first EOS that correctly models both critical behavior and vapor-liquid-liquid equilibrium (VLLE). The vapor-liquid equilibrium is described by a coexistence equation relating $T$ and $\mu$, which we may write conceptually in the form $T=T_{V L}(\mu)$. The degree of freedom lost by enforcing this coexistence relation reappears as an order parameter specifying the relative proportion of the vapor and liquid phases. Similarly, liquid-liquid equilibrium is described by a coexistence equation $T=T_{L L}(\mu)$, and an order parameter specifying the relative proportion of the two liquid phases. When the two coexistence surfaces meet, i.e., if $T=T_{V L}(\mu)=T_{L L}(\mu)$, we have exchanged two lost degrees of freedom by two order parameters specifying the relative proportion of the vapor and the two liquid phases. Now both the VL and the LL branch of the coexistence manifold must satisfy the equation $\Sigma(P, T, \mu)=0$. This is possible only if $\Sigma$ is nonlinear; cf. Anisimov et al. [2]. Already allowing $\Sigma(P, T, \mu)$ to be quadratic in $P$ suffices. However, in revised scaling models, $\Sigma$ is independent of $P$, and $P$ is uniquely determined by $T$ and $\mu$. This implies that in revised scaling only two simultaneous phases within the same phase group are possible, which excludes VLLE.

Returning to the general case, the critical exponents and the universal functions $s$ in (34) are independent both of the phase group and of the particular mixture. The freedom remaining, namely the form of the analytic expressions for the scaling fields, must be determined from the known the behavior at low density (virial equation of state) and any experimental information available. For phenomenological purposes we may choose all

\footnotetext{
${ }^{14}$ with the possible exception of multicritical points; cf. footnote ${ }^{4}$. However, tricritical points have classical critical exponents (Kortman [38], Riedel \& Wegner [51]), so they can probably be modelled (as for van der Waals fluids) by the analytic nonlinearities in the EOS. Only logarithmic corrections to scaling (StePhen et al. [55], WEGneR \& RiEDEL [60]) are neglected in such models, an approximation probably adequate in most applications.
} 
scaling fields as low degree polynomials or rational functions of $T, P$, and $\mu$ (or, as argued in Neumaier [44], of a reduced temperature, a reduced pressure, and reduced activities), and fit the coefficients to match experimental data. Simple restrictions discussed in [44] guarantee that, at low densities, one can deduce a virial equation of state with the correct multi-component structure.

To exploit the available freedom without incurring artifacts due to excessive parameter sensitivity, fitting procedures may make use of all techniques available for the construction of modern, accurate multiparameter equations of state, as reviewed, e.g., in LEMMON \& SPAN [40]. It may be expected that the result will be a global multi-component EOS that, by their very form, automatically has the following properties:

(i) Close to critical points, plait points, and consolute points, the correct universality and scaling behavior is guaranteed.

(ii) At low densities, one can deduce a virial equation of state with the correct multicomponent structure.

(iii) The Gibbs phase rule holds.

(iv) The equation of state makes globally sense, for arbitrary thermodynamic conditions in which only the phases modelled are present.

For binary mixtures, a global crossover EOS with correct critical scaling and a correct low density limit was first derived by KisElev \& FRIEnd [36], using the Schofield parameterization; see also KISELEV \& ELY [34]. The present approach is more general, needs no parameterization, and works for arbitrarily many components and phases.

\section{Conclusion}

Starting from generalized polar coordinates slightly more general than those used in the traditional Schofield-type parameterizations, we were able to choose the coordinates in such a way that the equation of state for the Ising universality class could be written in a parameter-free form. The resulting EOS expresses the square of the strong scaling field $\Sigma$ as an explicit function $\Sigma^{2}=D^{2 e_{-1}} W\left(D^{-e_{0}} \Theta, D^{-e_{1}} I_{1}, \ldots\right)$ of the dependent scaling field $D>0$, the thermal scaling field $\Theta$, and scaling correction fields $I_{1}, I_{2}, \ldots$, with a smooth, universal function $W\left(\theta, \iota_{1}, \ldots\right)$ and the universal critical exponents $e_{-1}=\delta /(\delta+1)$, $e_{0}=1 /(2-\alpha), e_{1}=-e_{0} \Delta_{1}, \ldots$

We deduced from the general form of the new EOS that - contrary to an in the past generally accepted belief - the dependent scaling field can be written as an explicit function of the relevant scaling fields without causing strongly singular behavior of the thermodynamic potential in the one-phase region.

With exact models, all parameterizations would lead to the same universal functions. However, different choices for the coordinatizing functions in the literature produce numerically very different parameterizations, and since all available models are approximate only, the universal functions computed from different models are slightly different. The EOS presented is the first one that only uses universal information (which encodes the 
underlying physics). A numerical expression for $W$ was derived, valid close to critical points.

We indicated how to use the new EOS to model multiphase fluid mixtures, in particular for vapor-liquid-liquid equilibrium (VLLE) where the traditional revised scaling approach fails. This is an important first step for the construction of industrial strength thermodynamic models for critical fluids and fluid mixtures of practical importance that are accurate over the whole experimentally accessible thermodynamic phase space.

\section{A Universality in generalized polar coordinates}

Because of the freedom in normalizing the scaling fields, a generalized polar coordinate system is universal only up to two positive scaling constants. Rescaling the basic fields according to the renormalization group transformation (7) with arbitrary $\lambda>0$ does not change the relations between the fields $D, \Sigma, \Theta$, and $\Omega$. Considerations of scaling may therefore assume without loss of generality that one of these fields is unchanged, which we take to be $\Theta$. Because of the definition of $\Omega$, this leaves two scaling degrees of freedom, given by the family of scale transformations

$$
\bar{D}=m h D, \quad \bar{\Sigma}=h \Sigma, \quad \bar{\Theta}=\Theta, \quad \bar{\Omega}=m \Omega
$$

The corresponding transformation of the parameterizing functions $c_{D}, c_{\Theta}, c_{\Sigma}$, and $c_{\Omega}$ is

$$
c_{\bar{D}}=m h c_{D}, \quad c_{\bar{\Sigma}}=h c_{\Sigma}, \quad c_{\bar{\Theta}}=c_{\Theta}, \quad c_{\bar{\Omega}}=m c_{\Omega}
$$

Using the traditional critical exponents (15), we find that the fractions

$$
\begin{gathered}
F:=|\Omega|^{-\delta} \Sigma, \quad G:=|\Theta|^{-\beta \delta} \Sigma, \quad K:=|\Theta|^{-\beta} \Omega, \\
X:=|\Omega|^{-1 / \beta} \Theta, \quad Y:=|\Sigma|^{-1 / \beta \delta} \Theta, \quad Z:=|\Sigma|^{-1 / \delta} \Omega,
\end{gathered}
$$

do not depend on the radial coordinate $r$, hence they are invariant under renormalization transformations. They scale by constant factors under scale transformations (35).

In an arbitrary generalized polar coordinate representation, we introduce the functions

$$
\theta(\xi):=c_{\Theta}(\xi), \quad \sigma(\xi):=\sqrt{\xi} c_{\Sigma}(\xi), \quad \omega(\xi):=\sqrt{\xi} c_{\Omega}(\xi)
$$

For models from the Ising universality class, $\sigma(\xi)$ and $\omega(\xi)$ are positive for $0<\xi<\xi_{\max }$, hence

$$
\operatorname{sign} \Omega=\operatorname{sign} \Sigma .
$$

This implies that, without loss of generality, we may restrict attention to states with $\Sigma \geq 0$. For such states, we find that the above fractions are functions

$$
\begin{gathered}
F(\xi):=|\omega(\xi)|^{-\delta} \sigma(\xi) \geq 0, \quad G(\xi):=|\theta(\xi)|^{-\beta \delta} \sigma(\xi) \geq 0, \quad K(\xi):=|\theta(\xi)|^{-\beta} \omega(\xi) \geq 0 \\
X(\xi):=|\omega(\xi)|^{-1 / \beta} \theta(\xi), \quad Y(\xi):=|\sigma(\xi)|^{-1 / \beta \delta} \theta(\xi), \quad Z(\xi):=|\sigma(\xi)|^{-1 / \delta} \omega(\xi) \geq 0
\end{gathered}
$$


Figure 3: The functions $f(\xi), g(\xi), k(\xi), x(\xi), y(\xi), z(\xi)$ for the Ising universality class in the $\Sigma$-explicit representation, where $\xi=\theta_{\max }-\theta$. The scaling constants of this representation are $F_{0}=1.162123$ and $X_{0}=0.617917$.
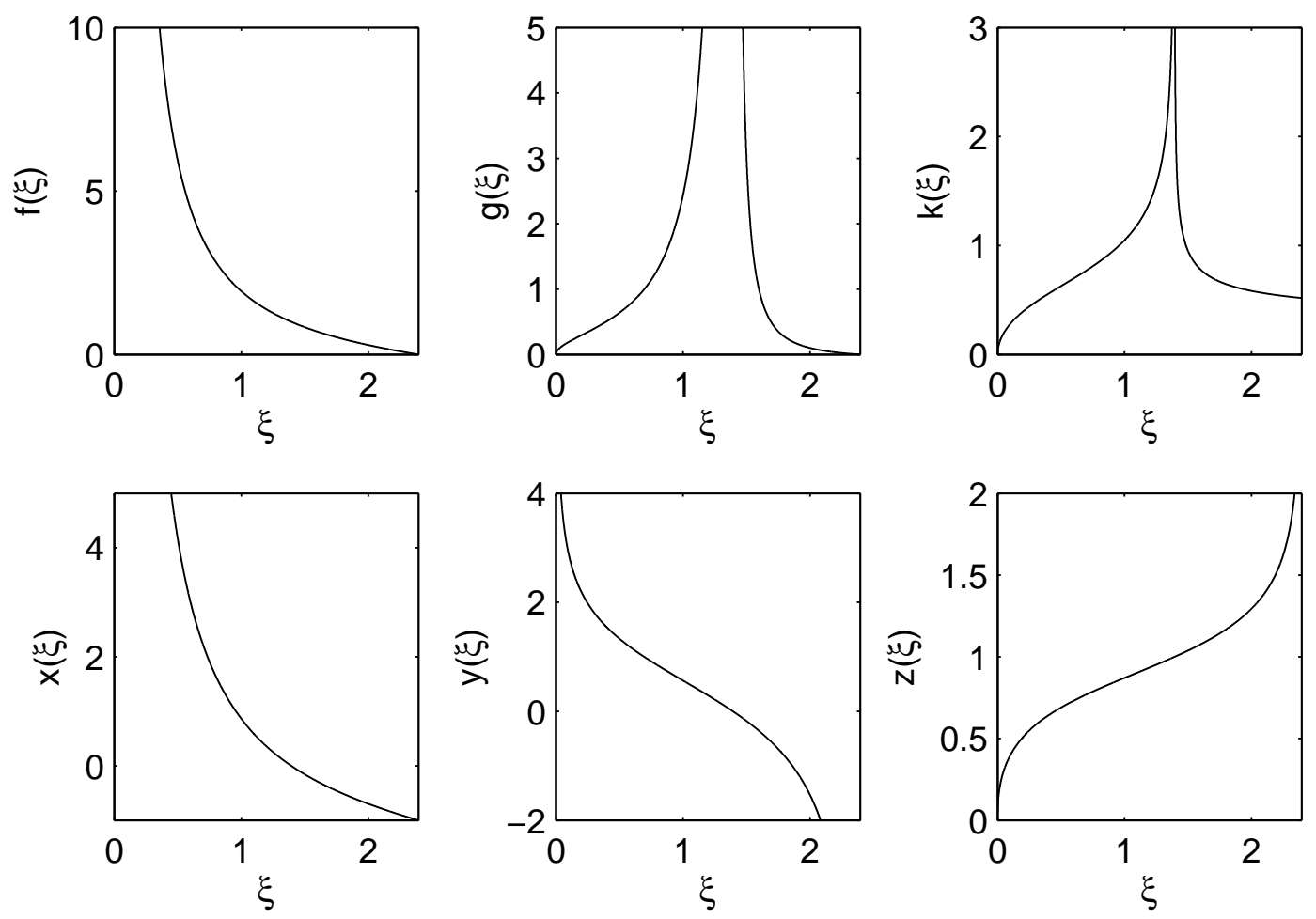

of $\xi$ that are analytic for $0<\xi<\xi_{\max }$. Only two of these functions are independent; expressed through $F$ and $X$, the other functions are given by

$$
G=F|X|^{-\beta \delta}, \quad K=|X|^{-\beta}, \quad Y=X F^{-1 / \beta \delta}, \quad Z=F^{-1 / \delta} .
$$

The two positive scaling constants

$$
F_{0}:=F\left(\xi_{0}\right), \quad X_{0}:=-X\left(\xi_{\max }\right)
$$

determine the scale of the generalized polar coordinates. Indeed, it is not difficult to see that with the additional scaling constants

$$
G_{0}:=F_{0} X_{0}^{-\beta \delta}, \quad K_{0}:=X_{0}^{-\beta}, \quad Y_{0}:=X_{0} F_{0}^{-1 / \beta \delta}, \quad Z_{0}:=F_{0}^{-1 / \delta}
$$

the quotients

$$
f:=F / F_{0}, \quad g:=G / G_{0}, \quad k:=K / K_{0}, \quad x:=X / X_{0}, \quad y:=Y / Y_{0}, \quad z:=Z / Z_{0}
$$

satisfy

$$
g=f x^{-\beta \delta}, \quad k=x^{-\beta}, \quad y=x f^{-1 / \beta \delta}, \quad z=f^{-1 / \delta},
$$

and are universal, since they are invariant under both the renormalization transformations and the scale transformations (35). For the $\Sigma$-explicit representation, they are drawn 
Figure 4: Universal relations between the asymptotic reduced fractions. Note that the graphs of $g_{+}(k)$ and $g_{-}(k)$ cross each other.
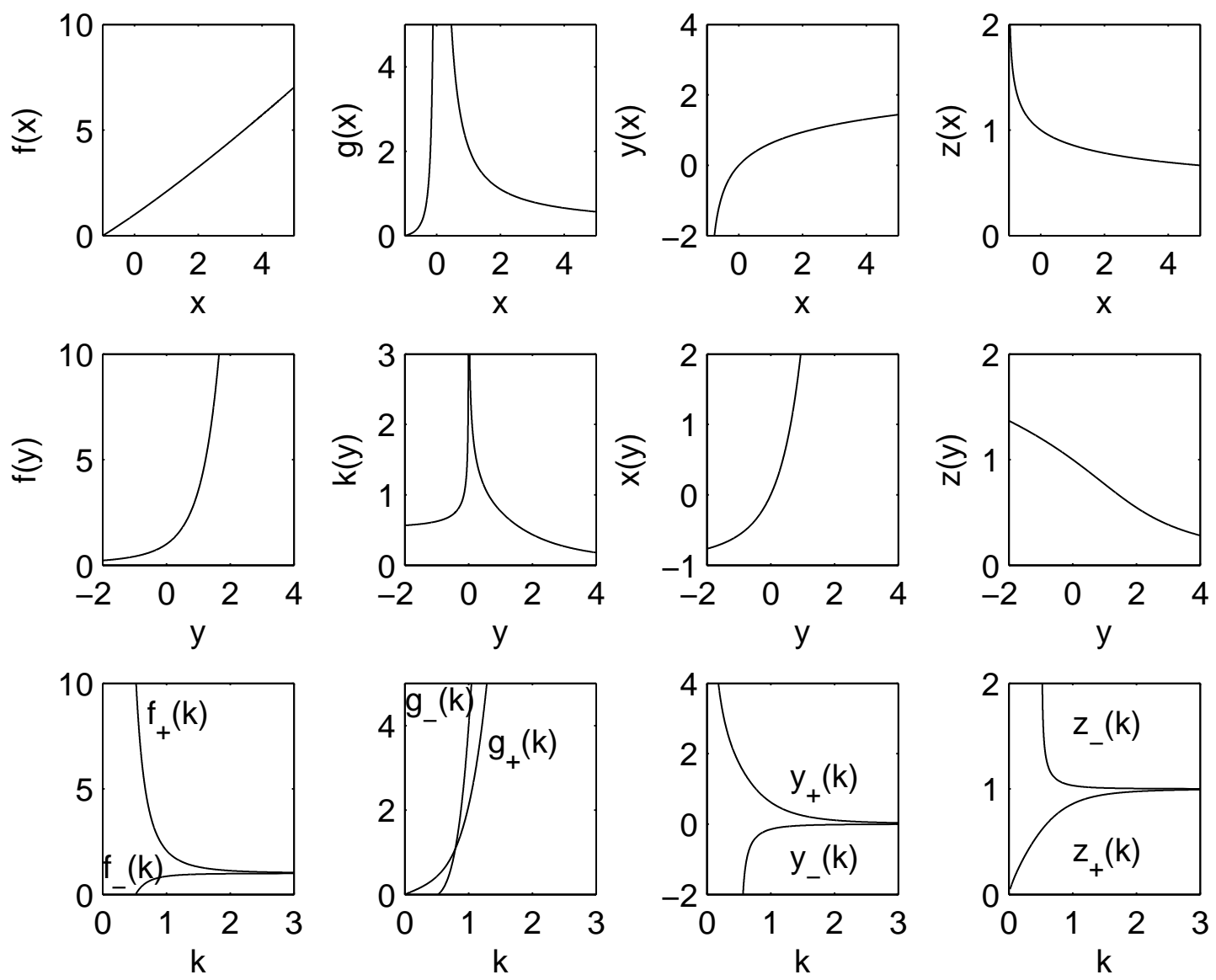

in Figure 3 in dependence on $\xi$. Since $x$ and $y$ are strictly monotone functions of $\xi$, we may express all quotients as functions of $x \in[-1, \infty]$ or $y \in[\infty, \infty]$ rather than $\xi \in\left[0, \xi_{\max }\right]$. Apart from (42), this gives the nontrivial relations

$$
\begin{aligned}
& F=F_{0} f(x), \quad G=G_{0} g(x), \quad Y=Y_{0} y(x), \quad Z=Z_{0} z(x), \\
& F=F_{0} f(y), \quad K=K_{0} g(y), \quad X=X_{0} x(y), \quad Z=Z_{0} z(y)
\end{aligned}
$$

(cf. Figure 4), involving universal analytic functions (denoted with a slight abuse of notation by) $f(x), g(x), y(x), z(x)$ and $f(y), g(y), x(y), z(y)$, respectively, expressing in different ways the same EOS relating $\Omega, \Sigma$ and $\Theta$. For example, $F=F_{0} f(x)$ becomes the $\operatorname{EOS}^{15}$

$$
\Sigma=F_{0}|\Omega|^{\delta} f\left(X_{0}^{-1}|\Omega|^{-1 / \beta} \Theta\right) .
$$

To compare more directly with microscopic data, we use the fact that $k(\xi)$, which has a singularity at $\xi=\xi_{0}$, is strictly monotone for $\xi<\xi_{0}$ (the high temperature regime $\Theta>0$ ) and for $\xi>\xi_{0}$ (the low temperature regime $\Theta<0$ ). Thus, for $\pm \Theta>0$, we

\footnotetext{
${ }^{15}$ Since $f(x)$ satisfies the normalization conditions $f(-1)=0$ and $f(0)=1$, this shows that $f(x)$ is identical with the universal function defined in Pelissetto \& ViCARI [46, (1.71)].
} 
may solve $k=k(\xi)$ for $\xi=\xi_{ \pm}(k)$ and express all quotients as functions of $k$ rather than $\xi$. This gives the trivial relation $x=k^{-1 / \beta}$ and the nontrivial relations

$$
F=F_{0} f_{ \pm}(k), \quad G=G_{0} g_{ \pm}(k), \quad Y=Y_{0} y_{ \pm}(k), \quad Z=Z_{0} z_{ \pm}(k)
$$

(cf. Figure 4), involving universal analytic functions $f_{ \pm}(k), g_{ \pm}(k), y_{ \pm}(k), z_{ \pm}(k)$. When $\xi$ is small, $k(\xi)=\xi^{1 / 2}(c+O(\xi))$ with a constant $c \neq 0$. This implies that $\xi_{+}(k)$ is an even analytic function of $k$ vanishing at $k=0$. This implies that we may write

$$
g_{+}(k)=g_{0} F_{H T}\left(k / k_{0}\right) \text { for } \Theta>0
$$

where

$$
F_{H T}(s)=\sum_{n=1}^{\infty} \frac{r_{2 n}}{(2 n-1) !} s^{2 n-1}
$$

is an odd universal function, ${ }^{16}$ which expresses the relation between $\Omega, \Sigma$ and $\Theta$ in the form of the high temperature EOS

$$
\Sigma=a|\Theta|^{\beta \delta} F_{H T}\left(b|\Theta|^{-\beta} \Omega\right) \quad \text { for } \Omega>0
$$

with $a=g_{0} G_{0}$ and $b=\left(k_{0} K_{0}\right)^{-1}$. In (43), $g_{0}$ and $k_{0}$ are universal scaling constants, chosen such that the coefficients of $F_{H T}(z)$ satisfy the normalization conditions

$$
r_{2}=r_{4}=1
$$

Values for the other coefficients $r_{2 k}$ of the high temperature expansion (44) have been computed from microscopic models by a number of different methods. BUTERA \& PERnICI [10, Table X] survey these methods and gives as presently most accurate values

$$
r_{6}=2.061(2), \quad r_{8}=2.54(4), \quad r_{10}=-15.2(4), \quad r_{12}=45(5), \quad r_{14}=1400(200) .
$$

(On the other hand, the universal analytic function $g_{-}(k)$ is not odd since $\xi_{-}(k)$ lacks the reflection symmetry of $\xi_{+}(k)$.)

\section{B Comparison with data from the literature}

Different generalized polar coordinate systems simply express these universal functions in different parametric forms. With exact models, all generalized polar coordinate systems would lead to the same universal functions. However, since all available models are approximate only, the universal functions computed from different models are slightly different. The traditional choice is based on arguments of simplicity, and uses

$$
c_{\Theta}(\xi):=1-\xi, \quad c_{\Omega}(\xi):=m_{0}
$$

\footnotetext{
${ }^{16}$ With $z_{0}:=k_{0}^{-1}$ and $F_{0}^{\infty}:=g_{0}^{-1} k_{0}^{\delta}$, (42) implies that $s=k / k_{0}=z_{0} x^{-\beta}$ and $s^{-\delta} F_{H T}(s)=F_{0}^{\infty} f(x)$. Hence $F_{H T}(s)$ is identical with the function called $F(z)$ in Pelissetto \& Vicari $[46,(1.84)]$.
} 
with a constant $m_{0}$ determined implicitly by the normalization $c_{\Sigma}(0)=1$. The resulting generalized polar coordinates have a qualitative geometrical meaning but are devoid of a clear physical meaning. (The same holds for the nontraditional choices discussed, e.g., in Fisher \& Zinn [21] and Pelissetto \& Vicari [46, Section 3.4].)

Indeed, different choices for the two coordinatizing functions may produce numerically very different parameterizations. Only the implicit functions defined by the parameterization (12) are (apart from scaling factors) universal as only they correspond to the underlying physics. For example, the numerical details of the forms reported in BUTERA \& PERNICI [10] and CAmpostrini et al. [14] are completely different, although they make very similar physical predictions and both start with the same coordinatizing functions ${ }^{17}(47)$. Only the nonuniversal constant $m_{0}$ in (47) is different, but this is enough to completely change the numerical coefficients: ButerA \& PERNICI [10, eq. (24)] report the numerical approximations $\varphi_{\max }=1.1273$ and

$$
c_{\Sigma}(\xi)=1-0.8014(50) \xi+0.00946(30) \xi^{2}+0.00141(40) \xi^{3}+0.00029(10) \xi^{4}-0.00011(5) \xi^{6} ;
$$

Campostrini et al. [14, Table V] report the numerical approximations $\xi_{\max }=1.37861$ (corresponding to $\varphi_{\max }=1.17414$ ) and

$$
c_{\Sigma}(\xi)=1-0.736743 \xi+0.008904 \xi^{2}-0.000472 \xi^{3} .
$$

(For other sources of numerical data, see AgAyAn et al. [1], CAmpostrini et al. [14], Guida \& Zinn-Justin [26], Hasenbusch [30].)

We checked that in the range plotted, the critical functions computed from the $\Sigma$-explicit EOS with $C(\theta)$ given by (26)-(27), differ from those computed from the (more recent and more accurate) parameterization by BUTERA \& PERnici [10, eq. (24)] by a root mean squared error of less than $2.5 \cdot 10^{-5}$. Indeed, the parameters were derived by numerical optimization of a measure of discrepancy between these functions.

\section{References}

[1] V.A. Agayan, M.A. Anisimov and J.V. Sengers, Crossover parametric equation of state for Ising-like systems, Phys. Rev. E 64 (2001), 026125.

[2] M.A. Anisimov, E.E. Gorodetskii, V.D. Kulikov and J.V. Sengers, Crossover between vapor-liquid and consolute critical phenomena, Phys. Rev. E 51 (1995), 1199-1215.

[3] M.A. Anisimov and J.V. Sengers, Critical region, Chapter 11 (pp. 381-434) in: Equations of state for fluids and fluid mixtures (J.V. Sengers et al., eds.), Elsevier, Amsterdam 2000.

\footnotetext{
${ }^{17}$ Butera \& Pernici [10] use $M=m_{0} R^{\beta} \theta, \tau=R\left(1-\theta^{2}\right)$, and $h=h_{0} R^{\beta \delta} \ell(\theta)$. This corresponds in the present notation to

$$
\Omega=m_{0} r^{\beta} \varphi, \quad \Theta=r\left(1-\varphi^{2}\right), \quad \Sigma=h_{0} r^{\beta \delta} \ell(\varphi),
$$

consistent with (17), though their constants $m_{0}$ and $h_{0}$ amount to a different scaling than that defining our $\Sigma$-explicit EOS. CAMPOSTRINI et al. [14] use essentially the same model but they write $h(\theta)$ in place of $\ell(\theta)$ and use a different value of $m_{0}$.
} 
[4] A. Bakhshandeh and H. Behnejad, Crossover parametric equation of state for asymmetric fluids, Chemical Physics 409 (2012), 32-36.

[5] A. Bakhshandeh and H. Behnejad, A new scaled crossover parametric equation of state for water, J. Iranian Chem. Soc. 10 (2013), 367-377.

[6] H. Behnejad, J.V. Sengers and M.A. Anisimov, Thermodynamic behaviour of fluids near critical points, Chapter 10 in: Applied thermodynamics of fluids (A.R. Goodwin et al., eds.), RSC Publ., Cambridge 2010.

[7] M.Y. Belyakov, S.B. Kiselev and J.C. Rainwater, Crossover Leung-Griffiths model and the phase behavior of dilute aqueous ionic solutions, J. Chem. Phys. 107 (1997), 3085-3097.

[8] C.E. Bertrand, J.F. Nicoll and M.A. Anisimov, Comparison of complete scaling and a field-theoretic treatment of asymmetric fluid criticality, Phys. Rev. E 85 (2012), 031131.

[9] P. Butera and M. Comi, Renormalized couplings and scaling correction amplitudes in the $N$-vector spin models on the sc and the bcc lattices, Phys. Rev. B 58 (1998), $11552-11569$.

[10] P. Butera and M. Pernici, Free energy in a magnetic field and the universal scaling equation of state for the three-dimensional Ising model, Phys. Rev. B 83 (2011), 054433 .

[11] J. Cai and J.M. Prausnitz, Thermodynamics for fluid mixtures near to and far from the vapor-liquid critical point, Fluid Phase Equilibria 219 (2004), 205-217.

[12] J. Cai, D. Qiu, L. Zhang and Y. Hu, Vapor-liquid critical properties of multicomponent fluid mixture, Fluid Phase Equilibria 241 (2006), 229-235.

[13] M. Campostrini, A. Pelissetto, P. Rossi and E. Vicari, Improved high-temperature expansion and critical equation of state of three-dimensional Ising-like systems, Phys. Rev. E 60 (1999), 3526-3563.

[14] M. Campostrini, A. Pelissetto, P. Rossi and E. Vicari, 25th-order high-temperature expansion results for three-dimensional Ising-like systems on the simple-cubic lattice, Phys. Rev. E 65 (2002), 066127.

[15] T.A. Edison, M.A. Anisimov and J.V. Sengers, Critical scaling laws and an excess Gibbs energy model, Fluid Phase Equilibria 150 (1998), 429-438.

[16] M.E. Fisher, Renormalization of critical exponents by hidden variables, Phys. Rev. 176 (1968), 257-272.

[17] M.E. Fisher, The renormalization group in the theory of critical behavior, Rev. Mod. Phys. 46 (1974), 597-616.

[18] M.E. Fisher and B.U. Felderhof, Phase transitions in one-dimensional clusterinteraction fluids IA. Thermodynamics, Ann. Physics 58 (1970), 176-216. 
[19] M.E. Fisher and B.U. Felderhof, Phase transitions in one-dimensional clusterinteraction fluids IB. Critical behavior, Ann. Physics 58 (1970), 217-267.

[20] M.E. Fisher and G. Orkoulas, The Yang-Yang anomaly in fluid criticality: experiment and scaling theory, Phys. Rev. Lett. 85 (2000), 696-699.

[21] M.E. Fisher and S.-Y. Zinn, The shape of the van der Waals loop and universal critical amplitude ratios, J. Phys. A: Math. Gen. 31 (1998), L629-L635

[22] D.S. Gaunt and C. Domb, Equation of state of the Ising model near the critical point, J. Phys. C 3 (1970), 1442-1461.

[23] R.B. Griffiths, Thermodynamic functions for fluids and ferromagnets near the critical point, Phys. Rev. 158 (1967), 176-187.

[24] R.B. Griffiths, Phase diagrams and higher-order critical points, Phys. Rev. B 12 (1975), 345-355.

[25] R.B. Griffiths and B. Widom, Multicomponent-fluid tricritical points, Phys. Rev. A 8 (1973), 2173-2175.

[26] R. Guida and J. Zinn-Justin, 3D Ising model: the scaling equation of state, Nuclear Phys. B 489 (1997), 626-652.

[27] K. Gutkowski, M.A. Anisimov and J.V. Sengers, Crossover criticality in ionic solutions, J. Chem. Phys. 114 (2001), 3133-3148.

[28] A. Hankey, T.S. Chang and H.E. Stanley, Tricritical points in multicomponent fluid mixtures, Phys. Rev. A 9 (1974), 2573-2578.

[29] M. Hasenbusch, Finite size scaling study of lattice models in the three-dimensional Ising universality class, Phys. Rev. B 82 (2010), 174433.

[30] M. Hasenbusch, Universal amplitude ratios in the three-dimensional Ising universality class, Phys. Rev. B 82 (2010), 174434.

[31] V. Holten, C.E. Bertrand, M.A. Anisimov and J.V. Sengers, Thermodynamics of supercooled water, J. Chem. Phys. 136 (2012), 094507.

[32] G.X. Jin, S. Tang and J.V. Sengers, Global thermodynamic behavior of fluid mixtures in the critical region, Phys. Rev. E 47 (1993), 388.

[33] Y.C. Kim, M.E. Fisher, and G. Orkoulas, Asymmetric fluid criticality. I. Scaling with pressure mixing, Phys. Rev. E 67 (2003), 061506.

[34] S.B. Kiselev and J.F. Ely, Simplified crossover SAFT equation of state for pure fluids and fluid mixtures, Fluid Phase Equilibria 174 (2000), 93-113.

[35] S.B. Kiselev and J.F. Ely, Generalized corresponding states model for bulk and interfacial properties in pure fluids and fluid mixtures, J. Chem. Phys. 119 (2003), $8645-8662$. 
[36] S.B. Kiselev and D.G. Friend, Cubic crossover equation of state for mixtures, Fluid Phase Equilibria 162 (1999), 51-82.

[37] S.B. Kiselev and J.C. Rainwater, Extended law of corresponding states and thermodynamic properties of binary mixtures in and beyond the critical region, Fluid Phase Equilibria 141 (1997), 129-154.

[38] P. Kortman, Scaling equation of state for thermodynamic systems having a tricritical point, Phys. Rev. Lett. 29 (1972), 1449-1452.

[39] C. Lamers and W. Schweika, Scaling of critical scattering in $\beta$-CuZn, Physica B: Condensed Matter 180 (1992), 326-328.

[40] E.W. Lemmon and R. Span, Multi-parameter equations of state for pure fluids and Mixtures, Chapter 12 in: Applied thermodynamics of fluids (A.R. Goodwin et al., eds.), RSC Publ., Cambridge 2010.

[41] S.S. Leung and R.B. Griffiths, Thermodynamic Properties near the Liquid-Vapor Critical Line in Mixtures of $\mathrm{He}^{3}$ and He${ }^{4}$, Phys. Rev. A 8 (1973), 2670-2683.

[42] P.H. Lundow and I.A. Campbell, Critical scaling to infinite temperature, Phys. Rev. B 83 (2011), 184408.

[43] L. Mistura, Critical phases in multicomponent fluid mixtures, J. Phys. A: Math. Gen. 9 (1976), 2139-2148.

[44] A. Neumaier, A multi-phase, multi-component critical equation of state, Manuscript (2013). arXiv:1307.8391 http://www.mat.univie.ac.at/ neum/ ms/critEOS.pdf

[45] K.E. Newman and E.R. Riedel, Critical exponents by the scaling-field method: The isotropic $N$-vector model in three dimensions, Phys. Rev. B 30 (1984), 6615-6638.

[46] A. Pelissetto and E. Vicari, Critical phenomena and renormalization-group theory, Physics Reports 368 (2002), 549-727.

[47] J.C. Rainwater, A nonclassical model of a type 2 mixture with vapor-liquid, liquidliquid, and three-phase equilibria, Int. J. Thermophysics 21 (2000), 719-737.

[48] J.C. Rainwater and D.G. Friend, Composition dependence of a field variable along the binary fluid mixture critical locus, Physics Lett. A 191 (1994), 431-437.

[49] J.J. Rehr and N.D. Mermin, Revised Scaling Equation of State at the Liquid-Vapor Critical Point, Phys. Rev. A 8 (1973), 472-480.

[50] , P.T. Reuter and K.A. Bugaev, Critical exponents of the statistical multifragmentation model, Phys. Lett. B 517 (2001), 233-238.

[51] E.K. Riedel and F.J. Wegner, Tricritical exponents and scaling fields, Phys. Rev. Lett. 29 (1972), 349-352. 
[52] P. Schofield, Parametric representation of the equation of state near a critical point, Phys. Rev. Lett. 22 (1969), 606-608.

[53] W. Schröer, A short history of phase transitions in ionic fluids, Contrib. Plasma Phys. 52 (2012), 78-88.

[54] J.V. Sengers and J.G. Shanks, Experimental critical-exponent values for fluids, J. Stat. Phys. 137 (2009), 857-877.

[55] M.J. Stephen, E. Abrahams and J.P. Straley, Logarithmic corrrections to the meanfield theory of tricritical systems, Phys. Rev. B 12 (1975), 256-262.

[56] P.M. Stevenson, Optimized perturbation theory, Phys. Rev. D 23 (1981), 2916-2944.

[57] M. Vicentini-Missoni, Equilibrium scaling in fluids and magnets, pp. 39-78 in: Phase Transitions and Critical Phenomena, Vol. 2 (C. Domb and M.S. Green, eds.), Acad. Press, New York 1972.

[58] H. Watanabe, N. Ito and C.K. Hu, Phase diagram and universality of the LennardJones gas-liquid system, J. Chem. Phys. 136 (2012), 204102.

[59] F.J. Wegner, Corrections to scaling laws, Phys. Rev. B 5 (1972), 4529-4536.

[60] F.J. Wegner and E.K. Riedel, Logarithmic corrrections to the molecular-field behavior of critical and tricritical systems, Phys. Rev. B 7 (1973), 248-256.

[61] J.C. Wheeler and R.B. Griffiths, Thermodynamic bounds on constant-volume heat capacities and adiabatic compressibilities, Phys. Rev. 170 (1968), 249-256.

[62] F. Zhong and M. Barmatz, Comparison of theoretical models of crossover behavior near the ${ }^{3}$ He liquid-vapor critical point, Phys. Rev. E 70 (2004), 066105.

[63] J. Zinn-Justin, Euclidean field theory and critical phenomena, 4th ed., Clarendon Press, Oxford 2002. 